\title{
Commentary
}

\section{Charles Twining}

Morris et al (2000) describe the varieties of current approaches to more detailed neuropsychological assessment in older people. How does this fit in with the reality of daily work for psychiatrists in general and old age psychiatrists in particular?

For some, perhaps many, simply the idea of having access to clinical psychology services may seem like an aspirational fantasy: the chance of even getting a referral seen would be a step forward. However, it is reasonable rather than aspirational to expect that any half-decent mental health service for older adults should certainly have an establishment for, and usually have recruited to, clinical psychology posts. They are of course unlikely to be plentiful and therefore need to be used wisely. How do you decide what to ask for and when?

Approaches to the assessment of cognitive impairment vary from observation (I once heard a junior doctor presenting a case in geriatric medicine include the observation that the patient "looked demented") to highly detailed neuropsychological examination with multiple forms of brain imaging.

I can recall the early days of computed tomography (CT) scanning, which was just beginning when I was a trainee clinical psychologist. Just for a brief while it seemed as though all that I had learned as an undergraduate psychologist would become redundant as the new imaging revealed all. Of course this did not happen then and has not happened yet. As Morris et al point out, neuropsychological assessment is about function rather than structure. However, the successive introduction of increasingly sensitive brain imaging techniques does give a useful model for how we might approach developing and using clinical psychology assessment skills.

Initially CT was rare, expensive and had to be justified at length for each patient, especially older ones. It is now seen as part of the standard work up for Alzheimer's disease. Perhaps functional magnetic resonance imaging (fMRI) will be like this in the future. For the moment you would ask for, say, a positron emission tomography (PET) or single photon emission computed tomography (SPECT) scan only if you thought it would add significantly to the picture.

The Mini Mental State Examination (MMSE) or something similar is routine. It works quite well for most cases, although it should be supplemented by systematic evaluation of activities of daily living, including those based on carer information and carer well-being. Requests for detailed neuropsychological assessment are for those cases where the picture does not quite add up. Typically, these include unusual presentations or cases where the screening assessment is at odds with other information, such as carers' or relatives' reports. One common example is when the patient has a premorbid IQ outside the normal range. More often the uncertainties arise for those with an IQ well above average. There are data that quite clearly show how the MMSE, for example, correlates with IQ (Christensen \& Jorm, 1992). I well remember a retired professional who presented at a memory clinic with a MMSE score of 29/30 but with relatives' reports suggesting cognitive problems. The Object Learning Test of the Kendrick Battery has no correlation with IQ (Kendrick \& Watts, 1999) and it, like the relatives' reports, more accurately predicted the subsequent clinical course.

\section{Trends in care}

Thus far, regular reassessment of cognitive function in dementia has been a feature of research rather

Charles Twining is Head of Psychology Services at Whitchurch Hospital, Cardiff CF14 7XB; tel.: 0292033 6580; fax: 0292033 6133; e-mail: charles.twining@cdffcom-tr.wales.nhs.uk. 
than of routine clinical practice. However, the protocols introduced regarding the use of acetylcholinesterase inhibitors for Alzheimer's disease make it very clear that one should decide who are 'responders' to any particular treatment. The question of whether 'no change' means the treatment is working remains a real conundrum.

It is suggested that one should evaluate treatment over a period of a few months. Some data on the Cambridge Automated Neuropsychological Test Battery suggest that particular sub-tests may be sensitive to very early cognitive changes in Alzheimer's disease (Fowler et al, 1997). Is it possible that such techniques might pick up drug response over a shorter period of just a week or two? This would certainly facilitate treatment decisionmaking.

Evidence from work with stroke patients and their families suggests that giving families feedback based on neuropsychological assessment reduces carer strain levels. It seems at least a reasonable hypothesis, and accords with clinical experience, that this might also be true for other cognitive impairment.

The implications of the Bournewood judgement (R. v. Bournewood NHS Trust, 1998) continue to echo through mental health care. One type of request that seems to be increasing is for expert opinion in relation to mental competence. To quote the British Medical Association/Law Society Guidance (1995): "An assessment from a clinical psychologist ... may assist in giving a detailed, validated and systematic assessment of cognitive functioning". Again for most cases detailed assessment may not be required, but it should be there when it is. Changes in the law, particularly the Mental Health Act and the care of those with impaired mental capacity, are likely to require more systematic and detailed assessment.

\section{Conclusion}

These are exciting times to be working in old age psychiatry. We expect within the next year to see the publication of the National Service Framework for Older People. This will include lists of mental health services and services for people with dementia, as well as the National Institute for Clinical Excellence's appraisal of donepezil, rivastigmine and galantamine for Alzheimer's disease. These developments are against the continuing steady growth in the numbers of very elderly people, who are most likely to have physical and mental health needs.

The mental health care of older people is an especially multi-disciplinary endeavour, and we no longer question whether there should be a multidisciplinary team. The challenge now is to fund, recruit and build that team using its many skills to best effect for the well-being of older people and their families.

\section{References}

British Medical Association/Law Society (1995) Assessment of Mental Capacity: Guidance for Lawyers and Doctors. London: BMA Publications.

Christensen, H. \& Jorm A. F. (1992) Effect of premorbid intelligence on the Mini-Mental State and IQCODE. International Journal of Geriatric Psychiatry, 7, 159-160.

Fowler, K. S., Saling, M. M., Conway, E. L., et al (1997) Computerized neuropsychological tests in the early detection of dementia: prospective findings. Journal of the International Neuropsychological Society, 3, 139-146.

Kendrick, D. C. \& Watts, G. D. (1999) The Kendrick Assessment Scales of Cognitive Ageing. Windsor: NFER-Nelson.

Morris, R. G., Worsley, C. \& Matthews, D. (2000) Neuropsychological assessment in older people: old principles and new directions. Advances in Psychiatric Treatment, 6, 362-370.

R. v. Bournewood Community and Mental Health NHS Trust ex parte L (1998)AllER 319. 